\title{
Comparative Study on Does Energy Drink Effect on the Reaction Time?
}

\author{
Mashal Fatima*, Aimen Malik, Seerat Fatima, Muhammad Shafique \\ Riphah International University, I-14 campus, Islamabad, Pakistan. \\ * Corresponding author. Tel: +92-334-5094092; email: mashal.fatima@riphah.edu.pk \\ Manuscript submitted July 3, 2018; accepted December 8, 2018. \\ doi: 10.17706/ijbbb.2019.9.1.59-64
}

\begin{abstract}
Reaction time is the time delay between the sensory stimulus and initiation of a motor response to a stimulus. Reaction time provides the information about the cognitive performance under certain conditions .In this paper energy drink and nicotine effects on the reaction time are investigated. This study is designed to acquire reaction/ response time from young adults whom consumes energy drinks and smokes frequently. Chemicals like nicotine, caffeine, taurine and glucose affects our brain in the way that these chemicals act to decrease the reaction time and improve cognitive performance and motor response. Using means of Delta Time as features and through statistical analysis it is concluded that there is difference in response time but not significant enough $(p>0.05)$. After consumption the reaction time decreases slightly which cannot be considered significant to influence brain activity.
\end{abstract}

Key words: Energy drink, reaction time.

\section{Introduction}

The reaction time is fundamentally the time delay when a stimulus reaches brain through sensory neurons. After being processed by the brain it initiates motor response or reaction occurs. It is mostly in an order of $200 \mathrm{~ms}$ depending upon the cognitive conditions [1], as this process deals with the domains of perception and motor planning. Reaction time is the stimulus-response phenomenon which involves two key factors; reaction time and learning. Reaction time varies from person to person and situation to situation. Reaction time is the time delay between the initiation of the stimulus and response. The stimulus used in this study was an audio stimulus. The delay between initiation of the signal and responding is collectively due to the time for the audio sensory signal to reach the brain, the time required by the brain to process the sensory information and generate a motor response.

A neurotransmitter is a chemical messenger which enables neurotransmission which means that they transmit signals across a neural junction. The neurotransmitters bind with the receptors of post synaptic neurons and reduce the time for motor response. Chemicals like caffeine, nicotine, taurine and glucose does effect brain activity [2]. Caffeine (1,3,7trimethylxanthine) ingestion promotes an improvement in human performance [3]. The effects of caffeine on performance are linked to both central and peripheral mechanisms. The effect of caffeine on the central nervous system (CNS) is linked to a blockade of adenosine receptors, which prevents a decrease in neuronal activity and subsequent an increase in motor response and muscle recruitment [4]. Nicotine affects cognition and behavior [5]. In smokers and nonsmokers, it produces small improvements in finger-tapping rate, motor response on tests of reaction time. To elucidate 
the neuroanatomical substrates of the effects of nicotine on cognition and reaction time a test of Audio Reaction time (RT) was used. Taurine which is present in Energy drink [6], increases the electrical activity (signaling ability) in nerve cells through effects on calcium, a key element required for proper electrical function of neurons. Taurine can imitate the actions of certain neurotransmitters, thus favoring the reaction time. The high glucose level stimulates both central and peripheral nervous system which in turn can influence the initiation of response to a stimulus and reaction time. This paper investigates the effects of certain chemicals like nicotine, caffeine, taurine, and glucose. It is hypothesized that consumption of these chemicals which are present in energy drinks can stimulate particular brain neurotransmitters which decreases the reaction time. Reaction time is also improved or decreases by learning [7] so to minimize the error random intervals (110s) were used for data analysis. Study was designed to acquire response time before and after the energy drink consumption. Comparison between the genders were also made. Further effects of nicotine, caffeine, taurine and glucose were also evaluated between smokers and nonsmokers.

\section{Methodology}

\subsection{Subject}

Study was conducted on total of 130 subjects. 80 subjects ( 40 males, 40 females, Average age 20 years, SD $=2.9$ ) for energy drink effects while 50 students (all males, average age 20 years, $\mathrm{SD}=3.5$ ) were registered for Nicotine's effect. All subjects were young adults and student at university. This study was designed on researching the effects based on the frequent consumption of energy drink and Nicotine (smoking). The study was allowed by Ethics committee of Riphah International University (Pakistan). Healthy and cognitively normal control subjects without past or present neurological disorders volunteered for the study, and gave their consent in the study. All the subjects were requested not to consume nicotine or any caffeine containing drink or food and to get proper sleep of 6 to 8 hours. Subject was provided comfortable room to relax before experimental procedure.

\subsection{Experimental Procedure}

Readings from the subjects were recorded by following a protocol.

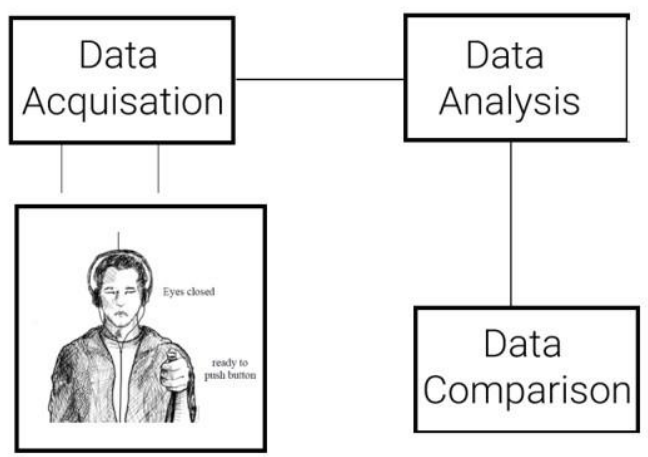

Fig. 1. Workflow of the proposed study starting from the data acquisition for reaction time, data analysis, comparison to find the trend between the chemicals.

The data for reaction time test was collected from the subjects using the BIOPAC $®$ Systems Inc. (USA) MP36 windows acquisition unit [8]. To provide anti-aliasing for the digital IIR filters and to reduce high frequency noise it employs an approximately $20 \mathrm{KHz}$ Low Pass Filter. Experimental procedure includes using a hand held push button (hand switch) as shown in Fig. 1, with dominant hand such that thumb is ready to push the button and headphones set properly. Subjects were asked to be seated and be relaxed 
with eyes closed. 10 random interval audio stimulus were given. At the instant the subject senses the auditory stimulus being triggered using BIOPAC, he/she has to press the push button in response to the stimulus.

Data was recorded before and after energy drink consumption. After consumption of energy drink namely, Red Bull, being popular among the university students [9], [10] data was again acquired with 30 minutes break in between. Same procedure was done for inquiring between the reaction time of smokers and non-smoker for evaluation of nicotine consumption. The rationale for measuring nicotine effects on reaction time only for male students was based on findings from Global Youth Tobacco Survey (GYTS) conducted in three cities of Pakistan which showed a very low prevalence of current smoking among female students [11].

\section{Data Analysis}

As seen in Fig. 2, when a subject pushes the hand switch a peak was recorded. Response time was calculated by using the Delta time $\Delta \mathrm{T}$. It is the difference in time between the end and beginning of the selected area, which is the duration of the selected area. The "selected area" is the area selected by the I-Beam tool (including the endpoints) as seen in Fig. 3. Ten random stimulus were triggered to minimize the error of learning. Delta time for every stimulus were calculated and then mean was obtained for every subject. Mean and standard deviation was calculated.

To validate the difference significance t-test was used. A statistically significant t-test result is one in which a difference between two groups is unlikely to have occurred because the sample happened to be atypical. For practical purposes statistical significance suggests that the two larger populations from which we sample are "actually" different.

\section{Results and Discussion}

After acquisition of reaction/response time before and after energy drink and cigarette consumption, data was compared both individually and between the groups.

There was a decrease in the auditory reaction time after the consumption of the energy drink. These changes induced and causing decrease in reaction time can be seen in Fig. 4. This is due to three ingredients of energy drink caffeine, taurine and glucose. Red bull consists of $40 \mathrm{mg}$ Caffeine in it. Caffeine [8], is a naturally-occurring, bitter, white, crystalline alkaloid. Adenosine is another alkaloid builds up in the brain throughout the day, which makes us feel tired when it get binds at adenosine receptors [9]. Caffeine is similar in structure with adenosine so it attaches with adenosine receptors and blocks adenosine to bind which increases our brain activity thus minimizing the reaction time.

Another component of energy drink is taurine .Taurine as reported by Goel et al. [12] improves nerve transmission; this means that movements made will be faster. The high glucose level increases Acetyl CoA, a precursor of Acetylcholine that stimulates both central and peripheral nervous system which in turn can influence the initiation of response to a stimulus and reaction time 13].

The Fig. 4, shows the decrease in the reaction time with the intake of nicotine. Nicotine is effective in decreasing the reaction time than energy drink. One of the ways that nicotine affects the brain is by mimicking neurotransmitters, nicotine resemble in shape with acetylcholine thus bind with its receptor. Nicotine can increase acetylcholine signaling throughout the brain which as a result increases reaction time [14]. Apart from chemical influence of energy drink composition and Nicotine in cigarettes, trend between the genders was also evaluated. The males have much less response time as compared to the females, but there is no significant difference. Females have $0.026 \mathrm{~s}$ slower reaction time then males. 


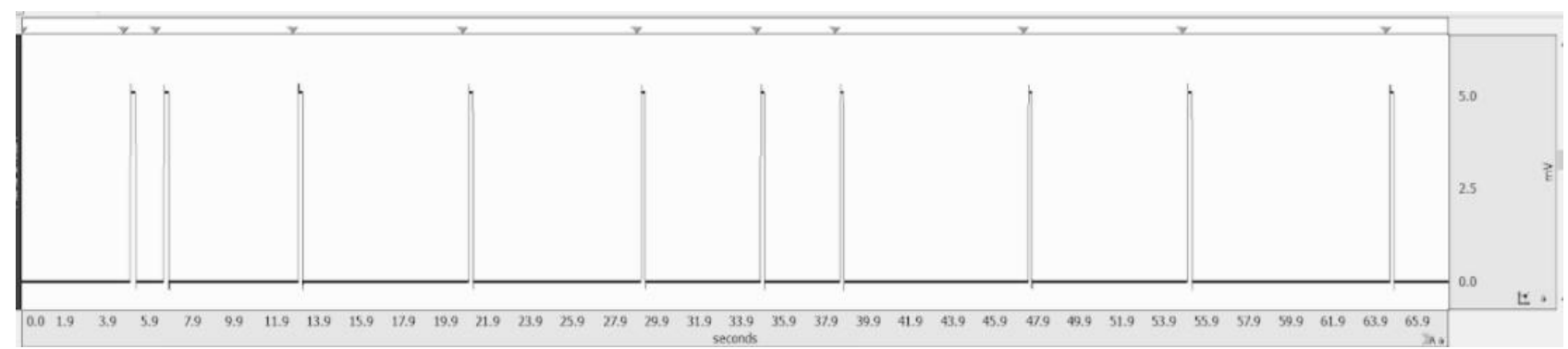

Fig. 2. Biopac window showing the response of the subject to the stimulus given at the random interval. When a subject listens to auditory stimulus, in response presses the push button. Clicks indicated by pulse and marker.

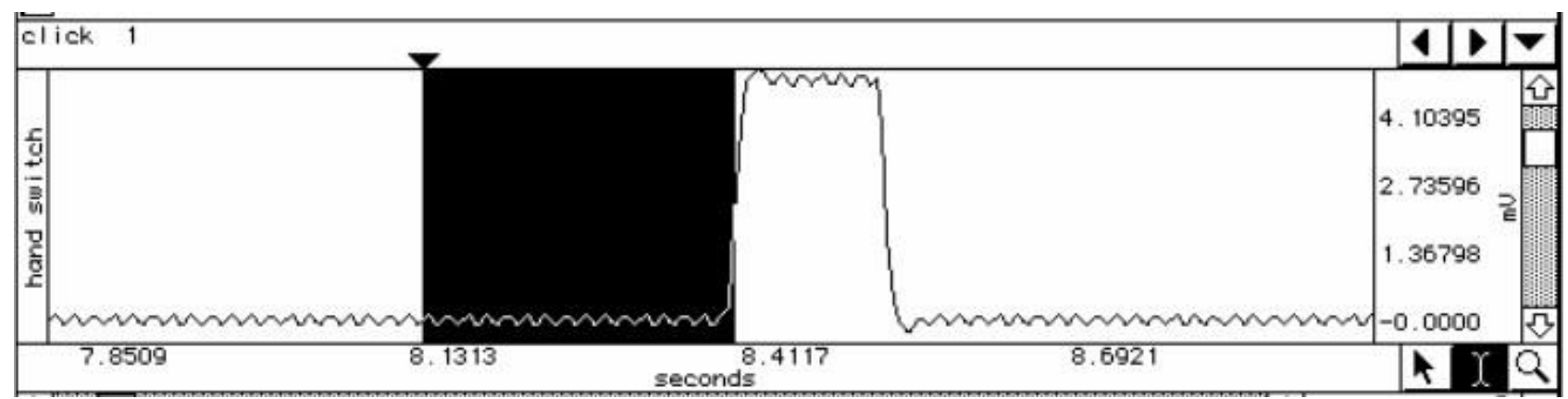

Fig. 3. Biopac window showing the response of the subject to the stimulus given at the random interval. Time delay to the response can be calculated by comparing the instant at which stimuli was triggered to the peak at which push button was pressed. The reaction time for this event is 0.265 seconds $(\Delta \mathrm{T})$.

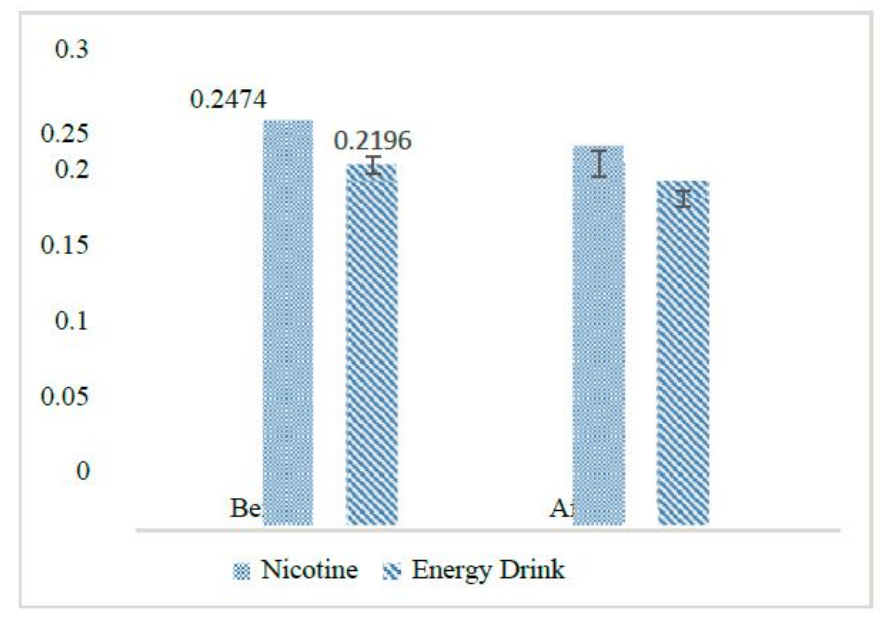

Fig. 4. Graphical representation of mean for Nicotine and energy drink (before and after). Decrease in reaction time is visually evident.

Man has strong motor response due to frequent use of motor pathway as they are involve in motor skills more than girls do and length of axon to a limb as average woman smaller then man Adam, Jos J. et al. [5].

The decrease was statistically not significant compared to its own baseline. As seen from Table. 1, there is change (decrease) in the mean of the response time when nicotine or energy drink is consumed, but the $p$ value is not less 0.05 . Inferring the results of student $t$-test it is concluded that energy drinks and nicotine does effect but their effect is not significant.

On contrast to the genders, it is evident from the means values that males does have low values or in other words prompt response to the stimulus as compared to the females. On applying the T-test for 
significance it is observed that the difference is not significant as shown in Table. 2.

Table. 1. Mean and Standard Deviation Observed Before and After the Energy Drink and Nicotine Consumption. Nicotine Consumers Are All Male Subjects

\begin{tabular}{lcll} 
Mean \pm SD & Before & After & P value \\
\hline Nicotine & $0.2474 \mathrm{~s}$ & $0.2196 \mathrm{~s}$ & 0.9410 \\
& \pm 0.0418 & \pm 0.033 & \\
Energy drink & $0.2311 \mathrm{~s}$ & $0.2088 \mathrm{~s}$ & 0.988 \\
& \pm 0.023 & \pm 0.016 & \\
\hline
\end{tabular}

Table. 2. Mean and Standard Deviation Observed between the Genders

Gender Male Female Pvalue

\begin{tabular}{cccc}
\hline Mean \pm SD & $0.255 \mathrm{~s}$ & 0.2816 & 0.064 \\
& \pm 0.359 & \pm 0.0391 & \\
\hline
\end{tabular}

\section{Conclusion}

Nowadays, Energy drink consumption and smoking is evident in young generation especially in university students. This study is aimed at investigating the effects of such energy drinks and nicotine in the students. Numerous studies have been concluded to study their adverse effects on health. This pilot study focus on the response time (cognition) of the young adults. By acquiring reaction time before and after of consumption and comparing them, it is concluded that energy drinks and nicotine does effect reaction time but this influence is not that significant. On other hand genders were compared too on the basis of their reaction time without any intervention, which shows that there is difference in response time but not significant enough.

\section{References}

[1] Wong, A. L., Adrian M. H., \& John W. K. (2015). Motor planning. The Neuroscientist, 21(4), 385-398.

[2] Robbbins, T. (2002). The 5-choice serial reaction time task: behavioural pharmacology and functional neurochemistry. Psychopharmacology, 163(3-4), 362-380.

[3] Inglis, F. M., Day, J. C., \& Fibiger, H. C. (1994). Enhanced acetylcholine release in hippocampus and cortex during the anticipation and consumption of a palatable meal. Neuroscience, 62(4), 1049-1056.

[4] Davis, J. K., \& Matt, G. J. (2009). Caffeine and anaerobic performance. Sports Medicine, 39(10), 813-832.

[5] Adam, J. J. (1999). Gender differences in choice reaction time: evidence for differential strategies. Ergonomics, 42(2), 327-335.

[6] Bazzucchi, I., et al. (2011). Caffeine improves neuromuscular function during maximal dynamic exercise. Muscle \& Nerve, 43(6), 839-844.

[7] Brice, C., \& Andrew, S. (2001). The effects of caffeine on simulated driving, subjective alertness and sustained attention. Human Psychopharmacology: Clinical and Experimental, 16(7), 523-531.

[8] Carmel, S., \& Alan, J. M. (2006). Physiological signal processing laboratory for biomedical engineering education. Proceedings of 27th Annual International Conference of the Engineering in Medicine and Biology Society, 2005. IEEE-EMBS 2005.

[9] Gillingham, R. L., Allan A. K., \& Peter T. (2004). Acute caffeine intake before and after fatiguing exercise 
improves target shooting engagement time. Aviation, space, and environmental medicine, 75(10), 865-871.

[10] Usman, A., \& Ambreen, J. (2012). Hypertension in a young boy: an energy drink effect. BMC Research Notes, 5(1), 591.

[11] Rozi, S., Zahid, A. B., \& Saeed, A. (2007). Correlates of cigarette smoking among male college students in Karachi, Pakistan. BMC public Health, 7(1).

[12] Goel, V., \& Manjunatha, S. (2014). Effect of red bull energy drink on auditory reaction time and maximal voluntary contraction.

[13] Seidl, R., et al. (2000). A taurine and caffeine-containing drink stimulates cognitive performance and well-being. Amino Acids, 19(3-4), 635-642.

[14] Malinauskas, B. M., et al. (2007). A survey of energy drink consumption patterns among college students. Nutrition Journal, 6(1), 35.

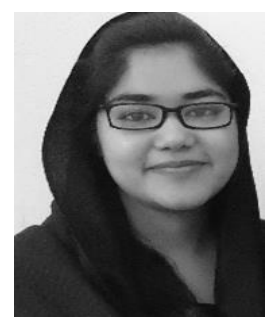

Mashal Fatima received the Ms. biomedical engineering degree from the Riphah international university (Awarded Gold Medal). Currently she is working as lecturer at same university. Her area of interest is in interdisciplinary research, which includes developing different feature extraction and classification algorithms for signal processing of different bio-signals for detection and rehabilitation, robotics interfacing and instrumentation.

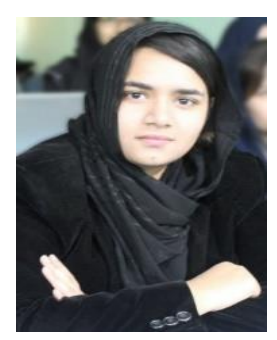

Seerat Fatima Jaffery is currently enrolled as a student at Riphah International University. She is studying the BSc. biomedical engineering. Her areas on interest include neural engineering and biomedical entrepreneurship.

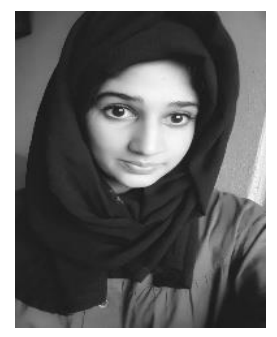

Aiman Malik is currently enrolled as a student at Riphah International University. She is studying the BSc. biomedical engineering. Her areas of interest include biomedical instrumentation and design and development of medical implants.

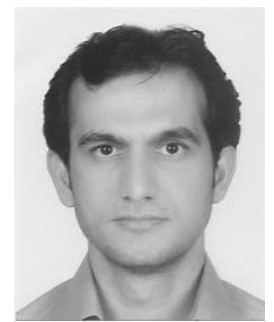

Muhammad Shafique is serving as head of biomedical engineering. He was awarded the PhD biomedical engineering degree in 2011 by City University London. He received his MSc degree in medical electronics and physics from Queen Mary University London in 2006 and B.E degree (receiving Gold Medal) in biomedical engineering from Sir Syed University of Engineering and Technology Karachi in 2003. During his PhD at City University London, he worked on novel techniques of photo plethysmography and pulse oximetry. His areas of interests are tissue optics, bioinstrumentation, and bio-signal processing. 